\title{
The corneal biomechanical changes after SMILE and LASIK refractive surgery were compared based on finite element analysis
}

\author{
Yinyu Song ${ }^{1}$, Lihua Fang ${ }^{1 *}$, Ruirui $\mathrm{Du}^{1}$, Luchao Lin $^{1}$, Xingming Tao ${ }^{1}$ \\ $1 *$ Key Laboratory of Nondestructive Test (Ministry of Education), Nanchang Hangkong University, Nanchang, 330063,86-18170938193, \\ fanglh71@126.com, China
}

\begin{abstract}
The three-dimensional (3D) finite element model of human eye was established, and the intraocular pressure (IOP) was loaded to simulate refractive surgery. The biomechanical properties of human cornea after SMILE and LASIK surgery were studied from the stress, strain and induced wavefront aberration. Our results showed that SMILE had less impact on the biomechanics, having less stress and strain changes than LASIK. However, the stress and strain of the cornea increased with the increase of the diopter and were concentrated in the central region. We also investigated the changes in wavefront aberrations of the cornea after surgery, and the results indicated that the defocus and vertical commotion were significantly affected by SMILE and LASIK surgery, while the remaining aberrations were approximately unchanged. In conclusion, both SMILE and LASIK sergury procedures changed the postoperative corneal biomechanics, but SMILE had less impact on the biomechanics of corneal.
\end{abstract}

\section{Introduction}

The rate of myopia is on the rise in China, which has become a prominent public health problem[1]. Myopia glasses allow people to see things, but other ways to treat myopia. Then there are also a growing number of treatments for myopia, including corneal refractive surgery, which allows myopic patients to remove their glasses. SMILE and LASIK refractive surgeryare a widely chosen method of refractive surgery. The main difference between SMILE surgery and LASIK refractive surgery is that SMILE surgery only requires a small incision to remove the corneal lenticule to complete the SMILE surgery[2].

The biomechanical properties of cornea have important reference significance, especially for the visual quality after refractive surgery. Abhijit et.al [3] found that the disadvantage of SMILE was that postoperative visual recovery was slower than LASIK. Kaiwei et.al [4] showed that corneal biomechanics were significantly decreased during refractive surgery. Guo et.al [5] studyed that SMILE was superior to LASIK in terms of biomechanical stability after surgeries. Osman et.al [6] also thought that LASIK and SMILE substantially decreased the corneal biomechanical properties, and the LASIK group had greater reduction. In addition, the cornea is the main refractive functional tissue of the eyeball[7], and it is important to maintain the completeness of corneal biomechanics[8].

Finite element analysis is a commonly used method to study ophthalmic surgery, which analyzes the mechanical characteristics of objects by mathematical methods [9]. Kaluzhny et.al [10] focused on the 3D corneal tissue models and the model studied in ophthalmic. Asher et.al [11] reviewed patient-specific modeling of the human cornea that can aid the clinician treatment of disease. Yan-Hui studied the mechanics application of the bovine eye finite element model [12]. In a word, the finite element analysis is very useful for the analysis of ophthalmic diseases. In our study, the biomechanics of cornea after SMILE and LASIK were compared and analyzed by establishing a 3D finite element model of the human eye.

The objective of this study is to construct a human eye model using finite element analysis to simulate conventional refractive surgery. The biomechanics of cornea were studied in terms of postoperative corneal stress, strain and induced wavefront aberration after SMILE and LASIK refractive surgery.

\section{Methods}

\subsection{The finite element model of human eye}

The siemens NX (Siemens AG, Munich, Germany) was used to build the model of human eye, which consisted of cornea and sclera in our model. And the geometrical structure size of the eye model was as follows: the radius of anterior surface curvature of the cornea was $7.7 \mathrm{~mm}$; the radii of posterior curvature of corneal was $6.8 \mathrm{~mm}$; The corneal central thickness and refractive index were $0.5 \mathrm{~mm}$ and 1.376, respectively. In addition, the boundary conditions of the model were that a fixed

\footnotetext{
Corresponding author: fanglh71@126.com
} 
constraint was placed at the bottom of the sclera and the cornea was trapped in the sclera. The eye model was as follows:

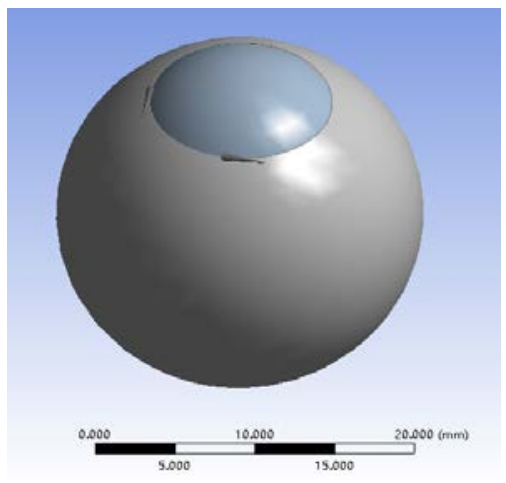

Fig. 1. The 3D whole-eye model

Due to different surgical methods, SMILE surgery only needed to make a small incision of $3 \mathrm{~mm}$ in the cornea surface, while LASIK made a corneal flap. At the same time, the mesh division of the model was also different in the ANSYS software. The cornea and sclera were divided by tetrahedral and hexahedral meshe in SMILE surgery, but they divided into hexahedral meshes in LASIK refractive surgery. Figure 2 showed the schematic diagram of different surgical eye models.
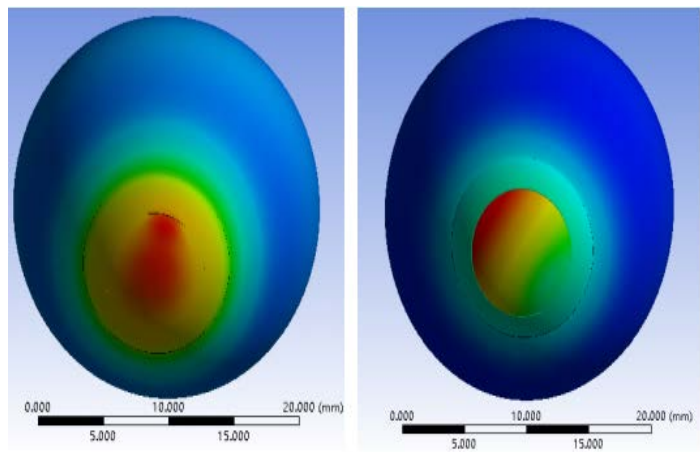

Fig. 2. (a) The SMILE surgical eye model. (b) The LASIK eye refractive surgery model. The black dotted line on the red area in figure $a$ is the small incision, and the colored ring in the center of image $b$ is the corneal flap.

\subsection{Material Properties}

Based on the cornea and sclera are anisotropic, hyperelastic and incompressible materials, we used the Ogden strain-energy function to set up the cornea and sclera materials in ANSYS. The specific expression is as follows:

$$
\mathrm{W}=\sum_{i=1}^{N} \frac{\omega}{\psi_{i}}\left(\bar{\lambda}_{1}^{\psi_{i}}+\bar{\lambda}_{2}^{\psi_{i}}+\bar{\lambda}_{3}^{\psi_{i}}-3\right)+\sum_{\mathrm{j}=1}^{m} \frac{1}{d_{j}}(J-1)^{2 j}
$$

$\psi$ and $d$ are material constants.

Then, the shear modulus $\omega$ is defined by:

$$
\omega=\frac{1}{2} \sum_{i=1}^{N} \psi_{i} \omega_{i}
$$

The bulk modulus $\mathrm{J}$ is as follows:

$$
j=\frac{2}{d_{1}}
$$

In the process of material fitting, the convergence was the best when corneal fitting parameters was $\mathrm{N}=2$ and scleral fitting parameters was $\mathrm{N}=1$. And the corneal fitting parameters were as follows: $\mu 1=0.0034801 \mathrm{MPa}$, $\mathrm{a} 1=104.06, \mu 2=0.0034801 \mathrm{MPa}$, and $\mathrm{a} 2=103.94$. The scleral fitting parameters were as follows : $\mu 1=$ $0.030224 \mathrm{MPa}$, and $\mathrm{a} 1=182.73$.

\subsection{Simulation of the refractive surgery}

Assuming the cornea is approximately spherical, we created the corneal stroma according to the principle of refractive surgery. The depth of corneal lenticule can be obtained by Munnerlyn approximate formula:

$$
l=l_{1}+l_{2}+l_{3}
$$

Then, $l_{1}, l_{2}, l_{3}$ were defined by:

$$
\begin{aligned}
& l_{1}=\sqrt{\left(\sqrt{R_{i x}^{2}-x^{2}}+R_{i y}-R_{i x}\right)^{2}-y^{2}} \\
& l_{2}=\sqrt{R_{f}^{2}-x^{2}-y^{2}}-\sqrt{R_{f}^{2}-\left(O_{z} / 2\right)^{2}} \\
& l_{3}=R_{i x}-R_{i y}-\sqrt{R_{i x}^{2}-\left(O_{z} / 2\right)^{2}}
\end{aligned}
$$

the preoperative radii of curvature of the meridians of the anterior corneal surface are Rix and Riy. OZ was the $\mathrm{X}$-axis diameter of the optical zone. $\mathrm{Rf}$ is the postoperative radius of curvature, it was expressed as:

$$
R_{f}=\frac{1000(n-1) R_{i x}}{(n-1)+D_{s} R_{i x}}
$$

Ds and $\mathrm{n}$ were the refraction in diopters and the refractive index of human cornea.

\section{$3 \quad$ RESULTS AND DISCUSSIONS}

\subsection{The changed of corneal stress}

Loading the IOP, the stress changed on the surfaces of the cornea were calculated at intervals of $-2 \mathrm{D}$ from 0 to 15D. Figure 3 shows the curve of the relationship between the stress and myopia diopter from SMILE and LASIK.
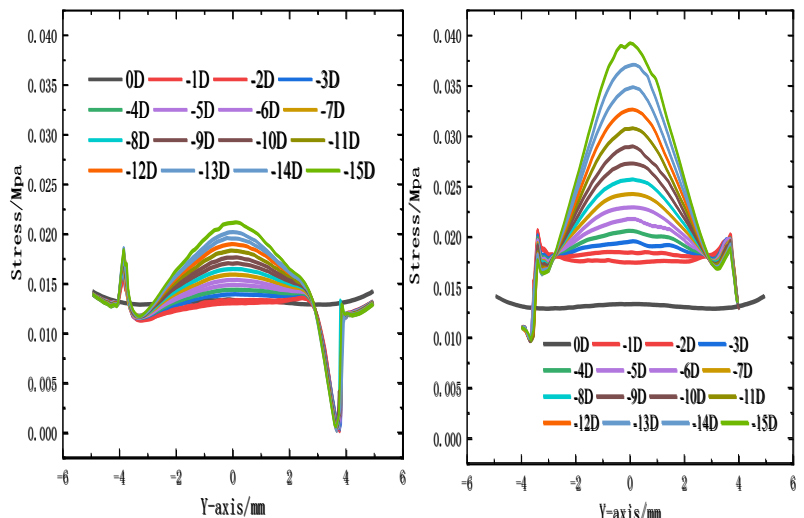

Fig. 3. (a) Relationship between stress and myopic diopter from SMILE surgery. (b) Relationship between stress and myopic diopter from LASIK refractive surgery. IOP was 15 $\mathrm{mmHg}$. The diameter of the optical zone was $6.6 \mathrm{~mm}$. 
On the whole, both Figure $3 \mathrm{a}$ and $3 \mathrm{~b}$ showed a regionalized distribution of stress in the central region, and it increased with the increase of diopter. When the diopter was $-15 \mathrm{D}$, the stress value in the central region of Figure $a$ and Figure $b$ reached the maximum. As could be seen in Figure 3a, the stress at $-3.8 \mathrm{~mm}$ and $3.8 \mathrm{~mm}$ on the $\mathrm{Y}$-axis had a tendency to protrude upward and downward, respectively. In $-3.8 \mathrm{~mm}$, the value of stress was $0.019 \mathrm{MPa}$, and the stress value is approximately 0 at $3.8 \mathrm{~mm}$. This phenomenon was caused by the construction of the corneal cap and cornea. It could also be seen from Figure $3 b$ that there was a jump at $-3.8 \mathrm{~mm}$ and $3.8 \mathrm{~mm}$ on the $\mathrm{Y}$ axis. Different from Figure $3 \mathrm{a}$, the latter all were small peaks upward, which stress values were $0.021 \mathrm{Mpa}$. In addition, we observed from the above results that the change of corneal stress after SMILE surgery was significantly smaller than the LASIK surgery.

\subsection{The changed of corneal strain}

We used the same method to calculate the corneal strain changes after SMILE and LASIK surgery under different myopic diopter, that the diopter ranged from -1D to $-15 \mathrm{D}$. The results were as follows.
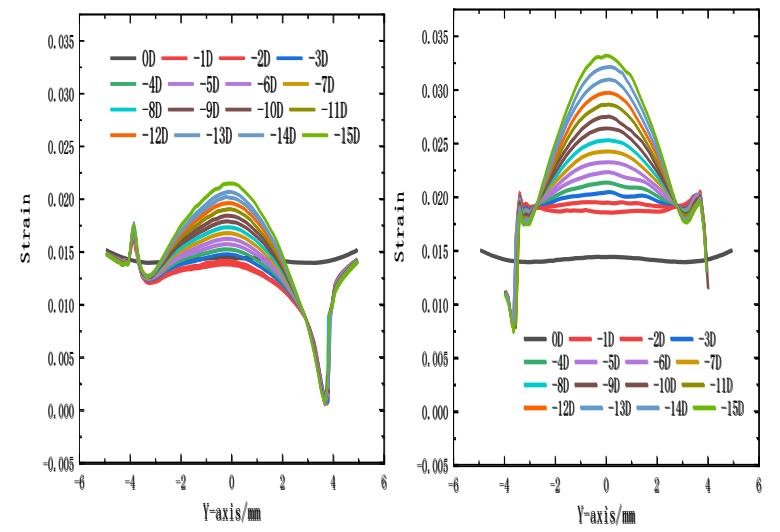

Fig. 4. (a) Relationship between strain and myopic diopter from SMILE surgery. (b) Relationship between strain and myopic diopter from LASIK refractive surgery. IOP was 15 $\mathrm{mmHg}$. The diameter of the optical zone was $6.6 \mathrm{~mm}$

In general, the strain after corneal surgery also increased with the increase of myopic diopter and was concentrated in the central region. According to Figure $4 \mathrm{a}$, a small peak appeared at $-3.8 \mathrm{~mm}$ with a value of 0.18 , and the sudden drop of strain value is approximately 0 at $3.8 \mathrm{~mm}$. Figure $4 \mathrm{~b}$ showed that there was a small peak at both $-3.8 \mathrm{~mm}$ and $3.8 \mathrm{~mm}$. And the all peak was caused by the fact that the cornea and the cornea cap or flap were constructed separated forms in the model components. In a word, with the increase of diopter, the change of corneal strain was more obvious, and LASIK had greater corneal strain after surgery.

\subsection{Induced wavefront aberration introduced by displacement of cornea}

In this part,we converted the displacement of corneal surface into the wavefront aberrations by wave surface fitting, investigated the changes of wavefront aberrations after SMILE and LASIK surgery. The result was shown in Figure 5.
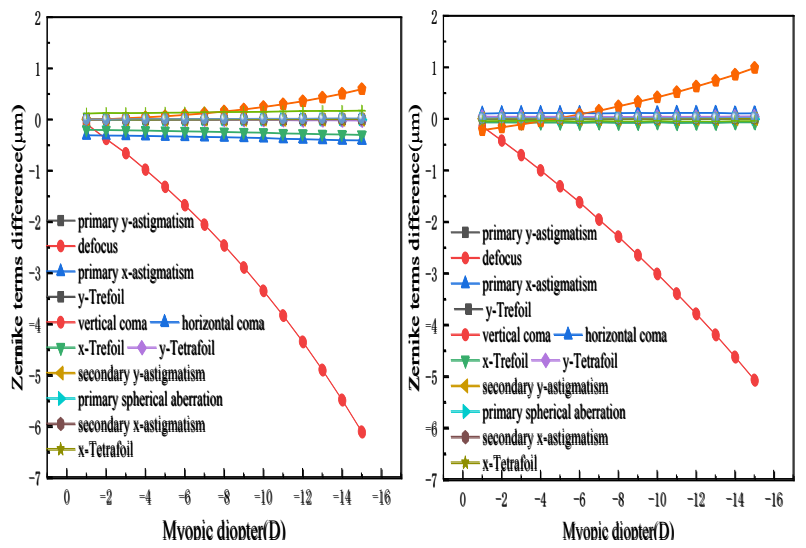

Fig. 5. (a) Relationship between induced wavefront aberrations on corneal surface and myopic diopter from SMILE (b) Relationship between induced wavefront aberrations on corneal surface and myopic diopter from LASIK. IOP was 15 $\mathrm{mmHg}$. The diameter of the optical zone was $6.6 \mathrm{~mm}$.

As could be seen from FIGURE 5, defocus and vertical coma changed the most, while other aberrations remain approximately unchanged. And compared with LASIK surgery, the defocus changed were more obvious with the increase of diopter after SMILE surgery, while the vertical coma changed were smaller. According to Figure 5a, The maximum values of defocus and vertical coma were approximately -6.2 and 0.6 , respectively. However, at the diopter of $-15 \mathrm{D}$, the value of defocus was about -5.3 and the value of vertical coma was about 1. In conclusion, the effects of SMILE and LASIK refractive surgery on defocus and vertical coma were significant, and the remaining wavefront aberration approximation did not change .

\section{DISCUSSIONS}

Corneal biomechanics is an important parameter to measure corneal tissue and can provide reference value for postoperative visual quality. Previous studies have compared the postoperative outcomes of SMILE and LASIK surgery, Majid et.al [13] showed that SMILE was efficient and safe, but the latest generation of LASIK surgery was better; Marcus et.al [14] thought SMILE may be a better refractive surgery and may replace LASIK in clinical practice. In this study, we studied the biomechanical changes of cornea after SMILE and LASIK refractive surgery by finite element analysis. And our result consistent with Hui's research, the biomechanics of SMILE surgery was superior to LASIK surgery. Although the clinical application of refractive surgery has achieved good results, there are still many areas to be improved. In order to better evaluate the biomechanical properties of the cornea after surgery, Our next work is to establish a $3 \mathrm{D}$ finite element model of the human eye that is closer to the human eye, to study the biomechanical of cornea after 
surgery, and to provide better theoretical knowledge for the clinical application of ophthalmology.

\section{CONCLUSIONS}

A 3D finite element model of the human eye was established to simulate SMILE and LASIK refractive surgery. And we compared the biomechanical changes of cornea after SMILE and LASIK refractive surgery from three aspects: stress, strain and induced wavefront aberrations. The results showed that the stress and strain of corneal changes presented regional concentration and increased with the increase of corrected diopter, but the postoperative stress and strain changes of SMILE were smaller than those of LASIK. In addition, the results also showed the changes of cornea induced wavefront aberrations, in which SMILE and LASIK surgery had significant effects on defocus and vertical coma. And SMILE surgery had greater defocus changes than LASIK, but less vertical coma changes. In summary, SMILE and LASIK refractive surgery had influence on the biomechanics of corneal.

\section{References}

1. $\mathrm{Xu}, \mathrm{X} ., \mathrm{S} . \mathrm{Li}, \mathrm{Y} . \mathrm{Xu}$, and J. Wei, Prevalence of myopia among primary school students in mainland China: a Meta-analysis. Guoji Yanke Zazhi: p. 1221-1227. [2016].

2. Kim, A. and R. S. Chuck, Wavefront-guided customized corneal ablation. Current Opinion in Ophthalmology, 19(4): p. 314-320. [2008].

3. Abhijit, Sinha, Roy, William, J., Dupps, et al., Comparison of biomechanical effects of smallincision lenticule extraction and laser in situ keratomileusis: Finite-element analysis. Journal of Cataract \& Refractive Surgery: p. 971-80. [2014].

4. Cao, K., L. Liu, T. Yu, F. Chen, J. Bai, and T. Liu, Changes in corneal biomechanics during smallincision lenticule extraction (SMILE) and femtosecond-assisted laser in situ keratomileusis (FS-LASIK). Lasers in Medical Science, 35(3): p. 599-609. [2020].

5. Guo, H., S. M. Hosseini-Moghaddam, and W. Hodge, Corneal biomechanical properties after SMILE versus FLEX, LASIK, LASEK, or PRK: a systematic review and meta-analysis. BMC Ophthalmology, 19(1): p. 167. [2019].

6. Osman, I. M., H. A. Helaly, M. Abdalla, and M. A. Shousha, Corneal biomechanical changes in eyes with small incision lenticule extraction and laser assisted in situ keratomileusis. BMC Ophthalmology, 16(1): p. 123-123. [2016].

7. Dan, Z. R., T. J. Archer, and J. B. Randleman, Mathematical Model to Compare the Relative Tensile Strength of the Cornea After PRK, LASIK, and Small Incision Lenticule Extraction. Journal of Refractive Surgery, 29(7): p. 454-460. [2013].
8. Yueguo, C., Paying more attention on the effects of excimer laser corneal refractive surgeries on the corneal biomechanical integrality. Chinese Journal of Optometry \& Ophthalmology, 13(1): p. 577-579. [2011].

9. Zhao, F., X. Cao, N. Wang, and X. Chen, The research progress of finite element analysis in ophthalmology. Chinese Journal of Ocular trauma and occupational Eye Diseases 040(006): p. 477-480. [2018].

10. Kaluzhny, Y. and M. Klausner, In vitro reconstructed 3D corneal tissue models for ocular toxicology and ophthalmic drug development. In Vitro Cellular \& Developmental Biology - Animal, 57(2): p. 207-237. [2021].

11. Asher, R., A. Gefen, and D. Varssano, PatientSpecific Modeling of the Cornea, in Patient-Specific Modeling in Tomorrow's Medicine, A. Gefen, Editor. [2012], Springer Berlin Heidelberg: Berlin, Heidelberg. p. 461-483.

12. Cui, Y.-H., J.-F. Huang, S.-Y. Cheng, W. Wei, L. Shang, N. Li, et al., Study on establishment and mechanics application of finite element model of bovine eye. BMC Ophthalmology, 15(1): p. 101. [2015].

13. Moshirfar, M., M. S. Murri, T. J. Shah, S. H. Linn, Y. Ronquillo, O. C. Birdsong, et al., Initial Single-Site Surgical Experience with SMILE: A Comparison of Results to FDA SMILE, and the Earliest and Latest Generation of LASIK. Ophthalmology and Therapy, 7(2): p. 347-360. [2018].

14. Ang, M., D. Tan, and J. S. Mehta, Small incision lenticule extraction (SMILE) versus laser in-situ keratomileusis (LASIK): study protocol for a randomized, non-inferiority trial. Trials, 13(1): p. 75 . [2012]. 